\title{
Les naissances multiples dans les textes médicaux antiques
}

Véronique Dasen

\section{Summary}

Ancient medical writers and biologists elaborated different theories to explain the phenomenon of multiple births. The earliest extant texts are in the Hippocratic collection and in the physiological treatises of Aristotle. They express opposed ideas: for the Hippocratics multiple births are the result of an ideal conception, for Aristotle they are regarded as anomalies associated with notions of monstrosity and excess. These views shed light on ancient collective imagery. Three themes in particular are found in non-medical literature and iconography: twin birth as a model of ideal fecundity, the ambiguous status of twins of different sexes, and the relation of multiple births to monstrosity and animality, as evidenced by the motif of twins born from one egg.

\section{Résumé}

Les biologistes et médecins antiques ont élaboré différentes théories sur le phénomène des naissances multiples. Les textes les plus anciens se trouvent dans la Collection hippocratique et dans les traités de physiologie d'Aristote. Ils expriment des avis divergents: dans la tradition hippocratique, la gémellité apparaît comme le produit admirable d'une conception idéale, tandis qu'Aristote la considère comme une anomalie associée à des notions d'excès et de monstruosité. Ces théories apportent un témoignage précieux sur l'imaginaire collectif antique. Trois thèmes notamment apparaissent dans les textes non médicaux et dans l'iconographie: la gémellité comme modèle de fécondité idéale, le statut ambigu des jumeaux de sexes différents, les rapports de la multiparité avec la monstruosité et l'animalité, illustrés en particulier par le motif des jumeaux nés d'un œuf.

Véronique Dasen (Dr. Phil.), Le Hobelet, CH-1783 Pensier 
Les naissances multiples ont suscité l'intérêt de nombreux biologistes et médecins antiques, des philosophes présocratiques $\left(5^{\mathrm{e}} \mathrm{s}\right.$. av. J.-C.), à Oribase ( $4^{\mathrm{e}}$ s. apr. J.-C.) et Paul d'Egine ( $7^{\mathrm{e}}$ s. apr. J.-C.). La description de ce phénomène se trouve dans des textes de genres très variés: des exposés relatifs à l'anatomie et à l'embryologie, des ouvrages pratiques sur l'accouchement, ainsi que dans des listes d'observations cliniques accompagnées de commentaires plus généraux.

Les Anciens ont élaboré différents systèmes explicatifs qui rendent compte des conceptions gémellaires ordinaires (les jumeaux, de même sexe ou de sexes différents, sont engendrés lors d'un seul coït), et des conceptions gémellaires anormales (les jumeaux sont conçus suite à différentes relations sexuelles ou se développent de manière monstrueuse).

Les rapports de ces théories avec l'imaginaire collectif antique sont multiples. Trois thèmes en particulier apparaissent aussi dans les textes non médicaux et dans l'iconographie: le premier concerne la gémellité comme modèle de fécondité idéale, le second le statut ambigu des jumeaux de sexes différents, le troisième les rapports de la multiparité avec la monstruosité et l'animalité, illustrés notamment par le motif des jumeaux nés d'un œuf.

\section{Conceptions gémellaires ordinaires}

Les théories les plus anciennes et les plus complètes sont formulées dans la Collection hippocratique et dans les traités de physiologie d'Aristote. Ces théories sont importantes: elles seront reprises, sans modification majeure, durant toute l'antiquité grecque et romaine; elles vont même marquer profondément la réflexion médicale et l'imaginaire occidental jusqu'à nos jours. Les opinions des auteurs anciens sont empreintes d'ambivalence. Les avis sont partagés: dans la tradition hippocratique, la gémellité apparaît comme le produit admirable d'une conception idéale, tandis qu'Aristote la considère comme une anomalie associée à des notions d'excès et de monstruosité.

\section{La tradition hippocratique}

\section{La gémellité, modèle de fécondité idéale}

Dans la Collection hippocratique, deux textes s'attachent à élucider le mystère des conceptions gémellaires, le traité Du régime et celui De la nature de l'enfant, qui forme avec le livre De la génération le plus ancien traité d'embryologie conservé 1 . Ces textes donnent une image positive de la

1 Cf. R. Joly, Le niveau de la science hippocratique, Paris, 1966, 70-119. 
gémellité. La naissance de jumeaux y apparaît comme un phénomène rare, mais dans l'ordre naturel: il est déterminé par la structure de la matrice,

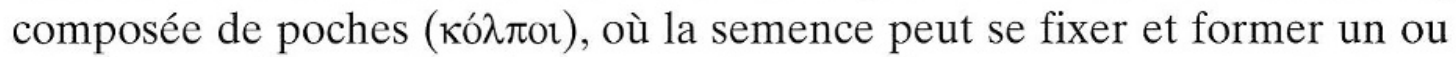
plusieurs embryons. Chez l'être humain ces poches sont au nombre de deux; elles sont disposées de manière symétrique de part et d'autre du col de la matrice $^{2}$. Mais chez les animaux multipares, comme la chienne ou la truie, leur nombre peut varier selon la fécondité de l'espèce, «les animaux qui portent beaucoup de petits en ont plus que ceux qui en portent peu» ${ }^{3}$.

La conception gémellaire ne peut avoir lieu que si des conditions optimales sont réunies: il faut que la matrice soit «développée semblablement des deux côtés de l'orifice, que l'ouverture soit égale et qu'elle soit sèche après les règles» ${ }^{4}$. Des jumeaux peuvent alors naître d'un seul coït si la sécrétion des deux partenaires est suffisamment abondante et vigoureuse pour se diviser et se développer dans chacune des deux poches.

On trouve les prémices de cette théorie des poches chez plusieurs philosophes de la nature. Démocrite semble être le premier à avoir expliqué que certains animaux comme la truie ou la chienne sont multipares parce qu'ils possèdent plusieurs matrices $(\mu \widetilde{\eta} \tau \rho \alpha$ ) et lieux ( remplir en plusieurs fois ${ }^{5}$. Mais seul l'auteur du traité Du régime explique clairement que la femme joue un rôle déterminant: pour que des jumeaux soient conçus, elle doit posséder une matrice bien conformée et émettre une semence d'importance égale à celle de son partenaire ${ }^{6}$.

2 Vict. 1, 30, 1 (Littré VI 504). Pour cette raison le terme qui désigne la matrice est généralement au pluriel $\left(\mu \widetilde{\eta} \tau \alpha_{1}\right)$ dans le Corpus hippocratique. L'auteur semble déjà connaître la théorie de l'utérus bicornis dont la trace la plus ancienne pourrait șe trouver chez Parménide; voir I. M. Lonie, The Hippocratic treatises "On Generation», "On the Nature of the Child», «Diseases $I V »$, Berlin/New York, 1981, 252-257.

3 Nat. puer. 31,1 (Littré VII 540).

4 Vict. 1, 30, 1 (Littré VI 504).

5 Elien, Nat. 12, 16 (= Diels, Vorsokr. 68 A 151); cf. Aristot. Probl. 892a 38-892b 3. La plus ancienne allusion à cette théorie des poches se trouve chez Phérécyde de Syros (Cronos remplit les cinq poches d'un utérus cosmique); Lonie (n. 2 supra) 255; H. S. Schibli, Pherecydes of Syros, Oxford, 1990, 16-19; A. E. Hanson, «Conception, gestation, and the origin of female nature in the Corpus Hippocraticum», Helios, 19, 1992,46-47. Un traité anonyme parle aussi de cinq poches: De generatione et semine in J. L. Ideler (éd.), Physici et medici Graeci minores, I, Berlin, 1841 (réimpr. Amsterdam, 1963), 296, section 20-21. Sur le concept d'un utérus à sept cellules au Moyen Âge, cf. R. Reisert, Der siebenkammerige Uterus. Studien zur mittelalterlichen Wirkungsgeschichte und Entfaltung eines embryologischen Gebärmuttermodells, Würzburg, 1986.

6 Vict. 1,30,1 (Littré VI 504). Sur la notion de semence féminine chez Hippocrate, L. A. DeanJones, Women's bodies in Classical Greek science, Oxford, 1993, 153-160. Dans la littérature médicale antique: H. Balss, «Die Zeugungslehre und Embryologie in der Antike. Eine Übersicht», Quellen und Studien zur Geschichte der Naturwissenschaften und der Medizin, 5, 1936, 35-40; E. Lesky, Die Zeugungs- und Vererbungslehren der Antike und ihr Nachwirken, Mainz, 1951 (AbhMainz 19, 1950); D. Gourevitch, «La gynécologie et l'obstétrique», in Aufstieg und Niedergang der römischen Welt, Berlin/New York, II, 37.3, 1996, 2099-2101. 
L'existence de ces deux demi-utérus démontre que la conception de jumeaux est dans l'ordre naturel. Les deux seins correspondent à ces deux poches. La Collection hippocratique contient plusieurs allusions à cette relation entre les seins et les deux parties de l'utérus. L'auteur des Aphorismes remarque qu'une femme enceinte dont les mamelles s'affaissent subitement est sur le point d'avorter ${ }^{7}$. Lorsque la femme est enceinte de jumeaux, l'aspect anormal d'un des seins annonce la fausse-couche d'un des enfants: «Une femme enceinte, portant deux jumeaux, si l'une des mamelles s'affaisse, avorte d'un des foetus; si c'est la mamelle droite qui s'affaisse, elle avorte du fœtus mâle; si la mamelle gauche, du fœtus femelle.» ${ }^{8}$

Cette correspondance se retrouve chez Galien ( $2^{\mathrm{e}}$ s. apr. J.-C.). Dans son traité De l'utilité des parties du corps humain, il déclare: «ce qui, j'en suis convaincu, dépassera à vos yeux tout ce qu'il y a de plus admirable, c'est que le nombre de cavités est égal à celui des mamelles»; il précise plus loin: «de même que la femme a deux cavités utérines qui aboutissent à un seul col, elle a deux mamelles qui sont comme les fidèles servantes, chacune de la cavité correspondante» ${ }^{9}$. Cette symétrie correspond à celle du corps humain tout entier, «composé de deux parties droite et gauche» ${ }^{10}$. Oribase en est si persuadé qu'il discerne des analogies de forme entre ces organes. Dans son traité Des parties génitales de l'homme, il explique que les prolongements latéraux de la matrice ressemblent à des seins de femme ${ }^{11}$.

L'existence d'un rapport entre le nombre de seins et la structure de la matrice est réaffirmé par des auteurs non médicaux. Plutarque loue lui-aussi le caractère providentiel de la nature: «sa prévoyance a sagement doté les femmes de deux seins pour que, même en accouchant de jumeaux, elles aient en double cette source de nourriture ${ }^{12}$. La nature a ainsi déterminé le nombre optimal d'enfants.

Ce discours semble correspondre à l'opinion commune, notamment dans le monde italique et gallo-romain. Diverses représentations d'époque romaine figurent une mère portant des jumeaux nouveau-nés qui symbolisent non seulement une maternité accomplie, mais la fécondité idéale. Une série de statuettes en terre cuite, fabriquées en Gaule centrale de la fin du I ${ }^{\mathrm{er}}$ s. apr. J.-C. au milieu du $\mathrm{III}^{\mathrm{e}}$ s. apr. J.-C., est particulièrement significative. Ces

7 Aph. 5, 37 (Littré IV 544). Cf. Superf. 19 (Littré VIII 486).

8 Aph. 5, 38 (trad. Littré IV 544-545).

9 De usu partium 14, 4 (Kühn IV 150), trad. C. Daremberg, I, Paris, 1854.

10 Ibid. Galien cite alors l'aphorisme 5,38 d'Hippocrate, répété dans le traité De locis affectis 6, 5 (Kühn VIII 437).

11 Collectiones medicae 24, 29 (éd. C. Daremberg/C. Bussemaker, III, Paris, 1858, 367).

12 Plut. Mor.3D. 


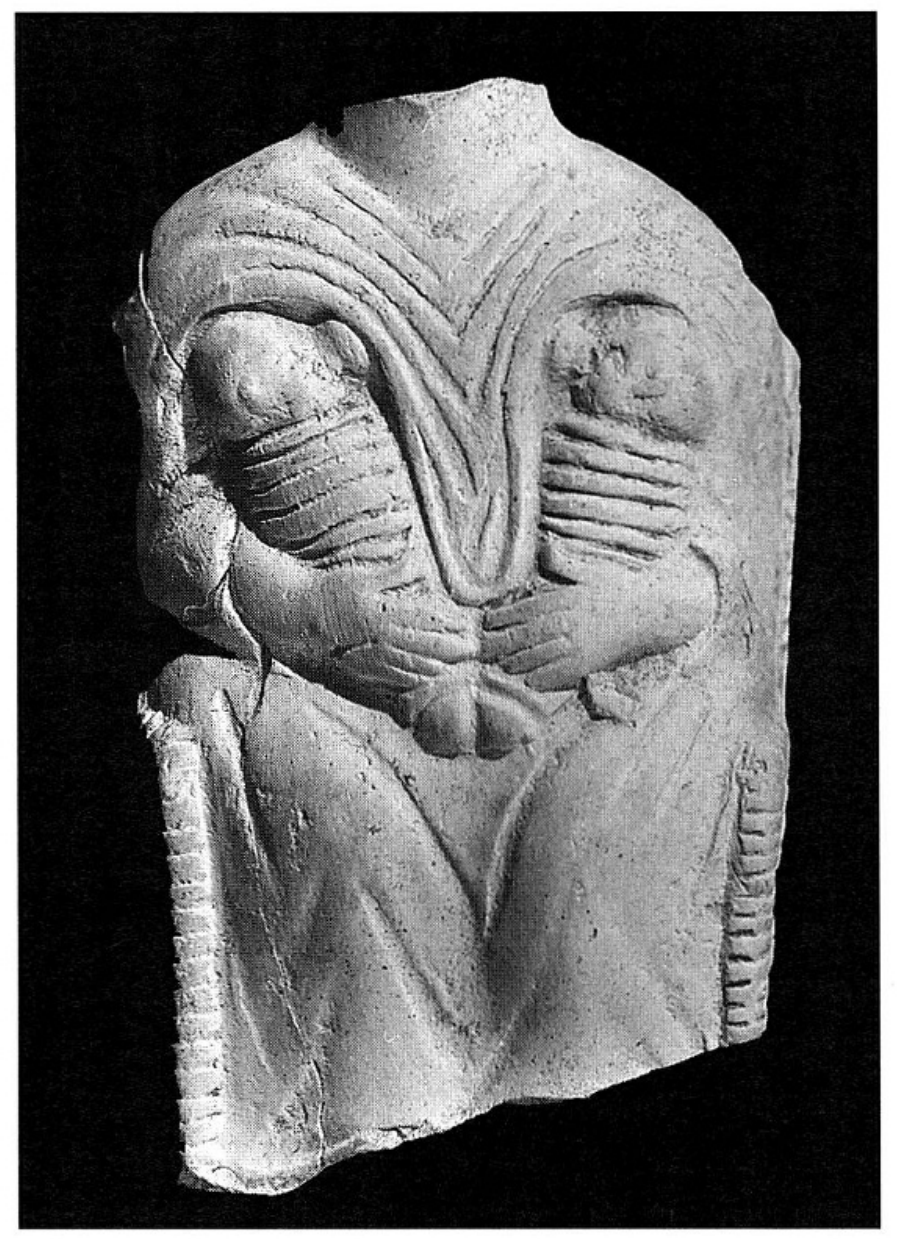

Fig. 1. Terre cuite. H. $8 \mathrm{~cm}$ (fin du $2^{\mathrm{e}} \mathrm{s}$. ap. J.-C.). Avenches, Musée romain, inv. 65/9595. Photo MRA.

figurines représentent une mère, assise dans un fauteuil en osier, en train d'allaiter deux bébés, un à chaque sein (fig. 1$)^{13}$. Ces objets semblent avoir rencontré une extraordinaire popularité. Leur aire de diffusion est très large; on les rencontre en quantité innombrable dans toute la Gaule, en Bretagne et dans les Germanies, jusqu'au limes et même parfois au-delà, en Germanie libre. Les trouvailles se répartissent entre les nécropoles, les habitats et, plus rarement, les sites cultuels, en particulier les sanctuaires des sources. Cette femme, conventionnellement appelée Dea nutrix, personnifie la Matrone par excellence; elle allaite des jumeaux qui symbolisent son inépuisable fécondité.

Cette image idéale de la maternité correspond au discours nataliste romain qui fait de la fécondité un véritable devoir civique. Elle apparaît à plusieurs reprises dans l'iconographie officielle, notamment sur un des panneaux de

13 V. Dasen, «A propos de deux fragments de Deae nutrices à Avenches: Déesses-mères et jumeaux dans le monde italique et gallo-romain», Bulletin de l'Association Pro Aventico, 39, 1997, 125-140. 


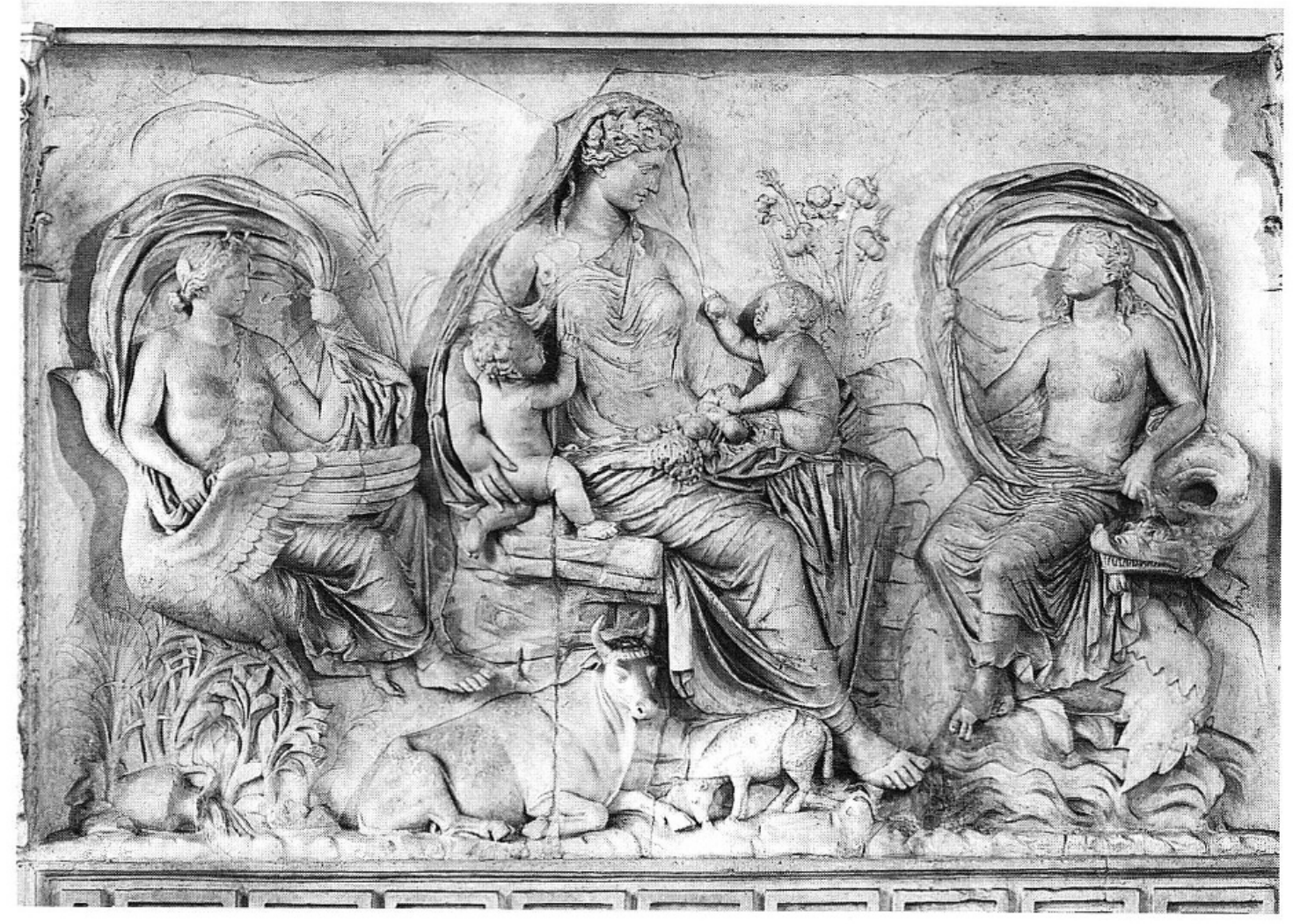

Fig. 2. Ara Pacis Augustae, Rome (13-9 av. J.-C.). Photo DAI, Rome, InstNegNr. 86. 1448.

l'Ara Pacis Augustae, cet autel monumental érigé en 13 av. J.-C. pour célébrer l'œuvre pacificatrice d'Auguste (fig. 2). La figure féminine centrale personnifie la Terre nourricière; elle est entourée de symboles de prospérité et de fertilité, des fleurs, des fruits, des animaux; deux jumeaux potelés sont assis dans son giron, gages d'abondance et de fécondité. Cette célébration de la gémellité est aussi présente dans le monnayage impérial. Marc-Aurèle fit ainsi frapper un sesterce pour commémorer la naissance, le 31 août 161, de ses fils jumeaux, le futur empereur Commode et son frère Antonin. Le type montre l'impératrice Faustine la Jeune en Felicitas debout, portant les jumeaux dans ses bras et entourée de ses quatre fillettes ${ }^{14}$.

La croyance en cette correspondance entre le nombre de seins et le nombre d'enfants traverse toute l'Antiquité classique; diverses anecdotes rapportées par J. Gélis montrent combien elle était encore ancrée dans l'opinion commune en Europe aux $17^{\mathrm{e}}$ et $18^{\mathrm{e}}$ siècles ${ }^{15}$.

14 Ibid. 133, fig. 14.

15 J. Gélis, L'arbre et le fruit. La naissance dans l'Occident moderne (XVI ${ }^{e}-X I X^{e}$ siècle), Paris, 1984,355 et 372 . 
Pour l'auteur du traité $D u$ régime, la simultanéité qui préside à la conception et à la naissance des jumeaux explique pourquoi ces enfants se ressemblent: «Les jumeaux se ressemblent pour les raisons que voici: tout d'abord, les lieux où ils se développent sont semblables, ensuite, ils ont été sécrétés en même temps; ensuite ils se développent à partir des mêmes aliments et enfin, ils viennent au jour en même temps. $\gg^{16}$ Un témoignage indirect signale que cette théorie continue d'être applicable après la naissance. Si les jumeaux grandissent dans les mêmes conditions (lieu, nourriture ...), leur état de santé peut évoluer de manière identique; Saint-Augustin rapporte ainsi que: «D'après Cicéron, Hippocrate l'illustre médecin a noté dans ses écrits qu'en voyant deux frères tomber malades et leur maladie en même temps s'aggraver et en même temps s'apaiser, il soupçonna qu'ils étaient jumeaux.» ${ }^{17}$

\section{Les jumeaux de sexes différents}

Mais si les jumeaux sont conçus lors d'un seul rapport sexuel, comment expliquer qu'ils puissent être de sexes différents?

Les auteurs hippocratiques ont déployé une grande ingéniosité pour rendre compte de ce phénomène. Ils proposent diverses explications. L'auteur du traité De la nature de l'enfant attribue la différence de sexes aux variations de qualité du sperme qui est émis en plusieurs fois: «la semence ne sort pas en une fois, mais est éjaculée en deux ou trois fois et il n'est pas possible qu'elle soit toujours également forte, la première à sortir et l'autre». Ces variations déterminent le sexe de l'enfant: «Dans la poche où est entrée la semence la plus épaisse et la plus forte, il se forme un garçon; dans celle où est entrée la semence la plus humide et la plus faible, il se forme une fille. Si une semence forte entre dans les deux, il se forme deux garçons; si c'est une semence faible, deux filles.» ${ }^{18}$

La théorie de l'auteur Du régime diffère. Chaque partenaire émet une semence tantôt mâle, tantôt femelle. Le sexe des jumeaux est déterminé par la prédominance de l'une de ces semences: «Quand la sécrétion des deux partenaires est mâle, il est fatal qu'un garçon naisse des deux côtés, et quand elle est femelle, une fille. Quand c'est une sécrétion femelle d'un côté et mâle de l'autre, celle qui l'emporte sur l'autre détermine le sexe.» ${ }^{19}$

16 Vict. 1,30, 2 (Littré IV 506).

17 Aug. Civ. 5, 2 (trad. G. Combès, Paris, Desclée de Brouwer, 1959); cf. Cic. Fat. 3, 5. Le sujet de la plaidoirie de Pseudo-Quintilien, Declamationes XIX maiores, 8 «Gemini languentes» (éd. L. Håkanson, Stuttgart, Teubner, 1982,151-174) concerne aussi une paire de jumeaux malades dont on pourrait sauver l'un en disséquant l'autre pour identifier leur mal.

18 Nat. puer. 31, 3 (Littré VII 540-542).

19 Vict. 1,30, 2 (Littré VI 504-506). 
Une autre théorie apparait dans plusieurs textes hippocratiques, celle de la latéralité. Le sexe de l'enfant peut être déterminé par le côté de l'utérus dans lequel il se forme: les embryons mâles se développent dans la partie droite, que l'on se représente chaude et sèche, les femelles dans la partie gauche, qui est à l'inverse froide et humide. Une variante imagine que c'est le testicule qui a produit la semence qui détermine le sexe de l'enfant: le testicule droit conçoit les garçons, le testicule gauche les filles. Il suffirait ainsi de se lier le testicule droit ou gauche pour engendrer un enfant de sexe féminin ou masculin ${ }^{20}$. Cette théorie associant la droite aux valeurs mâles et la gauche aux valeurs femelles est déjà énoncée chez Parménide et Anaxagore; elle est reprise par de nombreux auteurs antiques, comme Galien et Oribase $^{21}$.

On trouve aussi dans la Collection hippocratique des traces de la théorie d'Empédocle qui affirme que le sexe de l'enfant est déterminé par la chaleur de l'utérus: «Si les deux parties [de la semence] occupent des lieux également chauds, il naît deux garçons, si ce sont des lieux également froids, il naît deux filles, mais si l'un des lieux est plus chaud, l'autre plus froid, il y aura des naissances de sexes différents.» ${ }^{22}$

\section{Aristote}

\section{L'anomalie gémellaire}

Aristote consacre à la gémellité plusieurs discussions dans ses traités de physiologie. Comme Hippocrate, il explique que la conception de jumeaux peut résulter d'un seul rapport sexuel si la quantité de sperme émise en une fois est telle qu'en se partageant elle parvient à former plusieurs embryons. Il juge de même essentiel qu'il y ait «une certaine correspondance entre le résidu de la femelle et celui qui vient du mâle»; une surabondance de sperme uniquement entraîne la destruction par dessèchement de l'embryon ${ }^{23}$.

Aristote se distingue toutefois de ses prédécesseurs par sa conception tératologique de la gémellité. Dans la Génération des animaux, il classe la

20 Superf. 31 (Littré VIII 500). Cf. Aristot. Gen. an. 4, 1, 765a 23-5.

21 Cf. Plut. Mor. 905E et les critiques de Soranos, Gynaecia, 1, 15. Sur cette opposition droite/ gauche, voir G. E. R. Lloyd, "Right and left in Greek philosophy», in id., Methods and problems in Greek science. Selected papers, Cambridge, 1991, 27-48.

22 Cens. 6,10 = Diels, Vorsokr. 31 A 81 (trad. G. Rocca-Serra, Le jour natal, Paris, Vrin, 1980). Cf. Hippocr. Epid. 4, 21 et 6, 2, 25; Aph. 5, 38; Superf. 31. Sur ces différentes théories dans la littérature médicale antique, voir Balss (n. 6 supra) 67-70; Lesky (n. 6 supra); Dean-Jones (n. 6 supra), 166-170,192-193.

23 Gen. an. 4, 4,772a 4-22. 
production de jumeaux parmi les phénomènes qui relèvent de la monstruosité. Son raisonnement s'appuye sur deux constatations. Il observe premièrement que l'être humain est en principe unipare, comme tous les animaux de grande taille, chez qui seule une petite partie de la nourriture sert à produire un résidu séminal: «la nourriture passe chez eux à l'accroissement du corps» ${ }^{24}$. Une émission surabondante de semence est donc «une monstruosité, parce qu'elle se fait contre la règle générale et habituelle» ${ }^{25}$. Aristote note aussi qu'une grossesse multiple peut produire des monstres: «La multiplicité des petits empêche leur achèvement par une gêne mutuelle, et fait obstacle aux mouvements de la génération. ${ }^{26}$ Deux embryons, par manque d'espace, peuvent ainsi se souder dans l'utérus et former des jumeaux siamois. Aristote ne donne pas d'exemples chez l'homme mais il en cite quelques-uns dans le monde animal, notamment chez les ovipares. Aristote ne considère donc pas les naissances gémellaires comme le fruit d'une conception optimale. $\mathrm{Au}$ contraire il affirme que l'idéal est de ne produire qu'un seul enfant à la fois, parfaitement bien développé: «Voilà pourquoi ils (les monstres) sont moins fréquents chez l'homme. Car la plupart du temps cette espèce est unipare et met au monde un petit achevé: d'ailleurs, même chez l'homme, c'est dans les régions où les femmes sont multipares qu'il y a surtout des monstres, par exemple en Egypte. $»^{27}$

Alors que les traités hippocratiques ne parlent que de jumeaux, Aristote envisage les limites de la multiparité chez l'être humain. Dans l'Histoire des animaux, il fixe à cinq le nombre maximal d'embryons qu'une femme peut porter ${ }^{28}$. Cette observation est scientifiquement correcte: les quintuplés correspondent effectivement à la capacité physiologique maximale d'une femme. Au-delà de cinq embryons, les fœus naissent prématurément, faute de place, avant d'être viables ${ }^{29}$. Selon Strabon, Aristote aurait aussi signalé la naissance de septuplés en Egypte, mais on ne sait pas s'ils étaient vivants ni

24 Gen. an. 4, 4, 771a 27-29.

25 Gen. an. 4, 4, 772a 36-b 1.

26 Gen. an. 4, 4, 770b 24-27. Cf. Hippocr. Gen. 10, 2: le manque d'espace est une cause de malformation de l'embryon.

27 Gen. an. 4,4,770a 33-34. Cf.P.Louis, «Monstres et monstruosités dans la biologie d'Aristote», in J. Bingen/G. Cambier/G. Nachtergael (éds), Le monde grec. Hommages à Claire Préaux, Bruxelles, 1978, 276-284; R. Kent Sprague, «Metaphysics and multiple births», Apeiron, 20, 1987,97-102. Cette conception négative de la gémellité se retrouve chez Albert le Grand au $13^{\text {e }}$ s.; cf. J. M. Thijssen, «Twins as monsters: Albertus Magnus' theory of the generation of twins and its philosophical context», Bulletin of the History of Medicine, 61, 1987, 237-246.

28 Hist. an. 7, 4, 584b 33; cf. Gell. 10, 1.

29 Cf. E. Papiernik-Berkhauer/J.-C. Pons, Les grossesses multiples, Paris, 1991, 331. Autre cas de quintuplés mentionnés dans l'Antiquité: Gell. 10, 2 (sous le règne d'Auguste); Digesta Iustiniani 34, 5, 7 (sous le règne d'Hadrien); H. A., Vie d'Antonin le Pieux 9,3 (sous le règne d'Antonin le Pieux). 

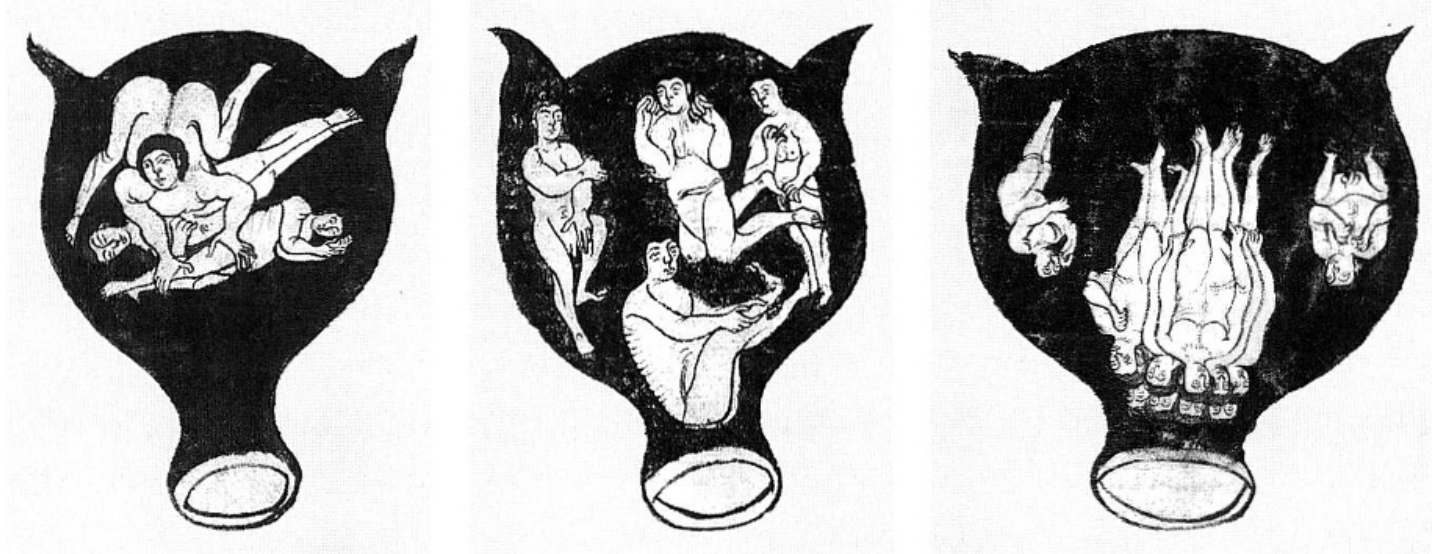

Fig. 3. Manuscrit de Moschion ( $9^{\mathrm{e}}$ s. apr. J.-C.). Bruxelles, Bibliothèque Royale, inv. 3701-15, folios 27 recto, 28 verso et 29 recto. Photo du Musée.

s'ils ont survécu ${ }^{30}$. L'Histoire des animaux évoque un cas encore plus exceptionnel: Aristote rapporte qu'une fausse-couche aurait entraîné l'expulsion de douze fœtus à la fois ${ }^{31}$. Ce cas illustrait peut-être le Traité d'obstétrique de Moschion ( $6^{\mathrm{e}} \mathrm{s}$. apr. J.-C.), une adaptation en latin du traité de gynécologie de Soranos $\left(1^{\text {er }} \text { s. apr. J.-C. }\right)^{32}$. Sur le plus ancien manuscrit de Moschion ( $9^{\mathrm{e}} \mathrm{s}$. apr. J.-C.), le texte est accompagné de dessins représentant l'enfant dans la matrice, figurée sous la forme d'un pot renversé avec deux sortes de cornes. Une série d'images montre des exemples de naissances multiples,notamment de triplés et de quadruplés ainsi que de douze fœtus bien groupés (fig. 3). Ni le texte de Moschion, ni celui de Soranos ne font référence à ce phénomène; l'illustrateur s'est peut-être inspiré de l'anecdote d'Aristote. Ce cas extraordinaire est théoriquement vraisemblable, puisque l'on a enregistré en 1971 la production de quinze fœetus chez une femme ayant subi un traitement inducteur d'ovulation ${ }^{33}$.

L'anomalie gémellaire peut aussi être démultipliée par une fécondité hors du commun.Dans l'Histoire des animaux, Aristote raconte qu'une femme mit au monde vingt enfants en accouchant à quatre reprises de quintuplés, et il

30 Strab. 15,1,22. Cf. Plin. Nat. 7, 33 (observation attribuée à l'historien Trogue Pompée).

31 Hist. an. 7, 4, 585a 10-12.

32 K. Sudhoff, Ein Beitrag zur Geschichte der Anatomie im Mittelalter, speziell der anatomischen Graphik, Leipzig, 1908 (réimpr. Hildesheim, 1964) 77, 79, pl. 23; Ch. Bonnet-Cadilhac, «Les représentations du fœtus in utero», Medicina nei secoli, 7.2, 1995, 339-350.

33 Le phénomène pourrait donc se produire naturellement; cf. Papiernik-Berkhauer/Pons (n. 29 supra) 334. Bonnet-Cadilhac (n. 32 supra) 348, n. 8 mentionne la représentation d'un utérus avec une vingtaine de fœtus sur un manuscrit latin du $14^{\mathrm{e}} \mathrm{s}$. 
ajoute que la plupart de ces enfants auraient survécu ${ }^{34}$. Le phénomène est également connu dans le monde animal. Aristote observe que certaines poules ne pondent que des œufs à double jaune ${ }^{35}$. Il note que cette particularité peut se transmettre héréditairement chez l'animal: «Les moutons et les chèvres donnent naissances à des jumeaux, quand ils ont une bonne nourriture, et si le bélier, ou le bouc, est lui-même né jumeau, ou si c'est le cas de la mère.» ${ }^{36}$ Asclépiade aurait lui-aussi attribué les conceptions multiples à la qualité exceptionnelle de certaines semences: «Selon Asclépiade, ils [les jumeaux et les triplés] proviennent des espèces différentes de sperme, comme les épis d'orge avec deux ou trois rangées de grains: il existe en effet des semences très fécondes. $»^{37}$ Mais aucun auteur ajoute que cette particularité peut se transmettre héréditairement au sein d'une famille. Seule la mythologie reflète cette réalité38: certaines familles légendaires comportent un nombre particulièrement élevé de jumeaux. L'exemple le plus frappant est celui de la descendance des jumeaux Agénor et Bélos: le roi Bélos engendra deux fils jumeaux, Danaos et Egyptos, et le fils d'Egyptos, Abas, eut deux fils jumeaux à son tour, Acrisios et Proetos. Sur l'ensemble de la descendance de cette «famille gémellaire», C. Voisenat a relevé pas moins de huit couples gémellaires sur dix générations ${ }^{39}$. De même des jumelles, les filles de l'Albain Sicinios, auraient chacune donné le jour à des triplés, les Horaces et les Curiaces, les légendaires héros de Rome et d'Albe ${ }^{40}$.

Aristote relève l'importance des facteurs géographiques et ethniques chez l'être humain. Il remarque que les femmes ont une fécondité anormalement élevée dans certaines régions du monde, notamment en Egypte ${ }^{41}$. Chez les Africains et les Egyptiens, «les naissances gémellaires sont courantes et pres-

34 Hist. an. 7, 4, 584 b 34-36. Cas également cité par Plin. Nat. 7, 33, qui le situe dans le Péloponnèse. Voir aussi Soranos, Gynaecia, 4, 1. Cf. l'exceptionnelle fécondité de l'impératrice Faustine la Jeune, mère de 13 enfants, dont à deux reprises de jumeaux (n. 14 supra).

35 Hist. an. 6,3,562a 27-29; Gen. an. 4, 3,770a 13-23; répété par Plin. Nat. 10, 146 (qui attribue cette observation à Celse).

36 Aristot. Hist. an. 6, 19, 573b 29-32. Colum. 3, 17 note que les bergers sélectionnent pour la reproduction les chèvres et les brebis qui mettent bas régulièrement des jumeaux et des triplés dans l'espoir «que la fécondité de la descendance correspondra à celle des parents».

37 Plut. Mor. 906B (= Ps.-Gal., De phil. hist. 32, Kühn XIX 326).

38 Cf. M. C. Phelan, «Twins», in R. E. Stevenson/J. G. Hall/R. M. Goodman (éds), Human malformations and related anomalies, Oxford, 1993, 1052-1053.

39 C. Voisenat, «La rivalité, la séparation et la mort. Destinées gémellaires dans la mythologie grecque», L'Homme, 105, 1988, 95-6 (fig.).

40 Dion. Hal. Ant. 3, 13, 3-4; Colum. 3, 8, 1 .

41 Hist. an. 7,4,584b 29-33; Gen. an. 4,4,770a 34-5. Autres régions: Aristot. Mir. 836a (1'Ombrie); ibid. 842 b (l'Illyrie, pour le bétail). 
que normales», répète Columelle ${ }^{42}$. L'origine de cette fécondité exceptionnelle est attribuée aux vertus des eaux du Nil. Pline note qu' «un chiffre supérieur [à trois jumeaux] passe pour un prodige, sauf en Egypte où les eaux du Nil sont un breuvage prolifique» ${ }^{43}$. Les auteurs anciens y localisent la plupart des records de naissances, comme celles de quintuplés ou de septuplés ${ }^{44}$. Même les chèvres y produisent des quintuplés d'après Elien ${ }^{45}$. Cependant les propriétés des eaux du Nil ont aussi des effets négatifs. Pour les auteurs classiques, l'Egypte est également la terre des monstres, humains et animaux ${ }^{46}$. Ce lien de l'Egypte avec les jumeaux a un aspect paradoxal. L'abondance des témoignages grecs et romains contraste avec l'extrême rareté des documents égyptiens d'époque pharaonique ${ }^{47}$.

\section{Les jumeaux de sexes différents}

Les jumeaux de sexes différents jouent un rôle important dans l'argumentation d'Aristote. Il les utilise pour réfuter les théories de ses devanciers sur la détermination des sexes, notamment celle de la polarité droite-gauche de Parménide et Anaxagore, et celle du froid et du chaud d'Empédocle. Les jumeaux de sexes différents constituent la meilleure preuve de l'incohérence de ces théories, puisqu'ils peuvent se former dans la même partie de l'utérus. Aristote se réfère à ses propres observations: «De plus, il arrive souvent que des jumeaux de sexes différents se forment en même temps dans la même partie de l'utérus: nous avons vérifié suffisamment le fait par les dissections opérées sur tous les vivipares, aussi bien terrestres qu'aquatiques», et plus loin: «on a vu une femelle dans la partie droite de l'utérus, un mâle dans la partie gauche, et les deux sexes réunis du même côté: le fait n'a pas été observé une seule fois, mais plusieurs.» ${ }^{48}$

42 Colum. 3, 8, 1. Certaines populations africaines au sud du Sahara ont effectivement des taux très élevés de gémellité. Au Nigéria, par exemple, un Yoruba sur onze est un jumeau. Le facteur ethnique et environnemental ne peut influencer que les grossesses dizygotes; cf. B. Pellegrini, «Les jumeaux dans le monde: biologie et culture», in C. Savary/Ch. Gros (éds), Des jumeaux et des autres, Genève, 1995, 52-54.

43 Nat. 7, 33. Cf. Strab. 15, 1, 22; Athen. 41e; Sén. Nat. 3, 25, 11. Sur les diverses propriétés des eaux du Nil pour les Anciens, voir D. Bonneau, La crue du Nil. Ses descriptions, ses explications, son culte, Paris, 1964, 104-109, 118-123.

44 Gell. 10,2,1; Strab. 15, 1, 22; Digesta Iustiniani 34, 5, 7.

45 Nat. 3, 33.

46 Cf. Aristot. Gen. an. 4, 4,770a 35.

47 Cf.J.Baines, in W. Helck/W.Westendorf (éds), Lexikon der Ägyptologie, Wiesbaden, VI, 1986, s.v. Zwilling, 1436-1437; J.-L. Chappaz, «Jumeaux, jumelles et «doubles〉 en Egypte ancienne», in Savary/Gros (n. 42 supra) 167-181.

48 Gen. an. 4, 1,764a; 765a 16-21. 
Aristote se démarque encore de ses prédécesseurs en considérant les jumeaux de sexes différents comme une catégorie à part. Leur naissance est particulièrement dangereuse: «Chez les humains, peu de jumeaux survivent si l'un est un garçon et l'autre une fille.» ${ }^{49}$ Pline le répète: «S'il naît deux jumeaux, il est rare que la mère ou que plus d'un bébé survive; si les jumeaux sont de sexe différent, il est encore plus rare qu'ils puissent être sauvés tous les deux.» $»^{50}$

Un préjugé très ancien veut en effet que les embryons mâles et femelles ne se développent pas de la même manière. Empédocle aurait déjà affirmé que le fœtus mâle arrive plus rapidement à terme que le fœtus femelle ${ }^{51}$. Divers auteurs apportent des précisions à l'appui de cette idée reçue; l'auteur du traité De la nature de l'enfant soutient que la fille est formée en 42 jours, le garçon en 30 jours $^{52}$. Aristote insiste lui-aussi beaucoup sur ces différences physiologiques entre les sexes: «chez l'homme, il y a plus de mâles que de femelles à naître avec des défectuosités (...) le mâle diffère beaucoup de la femelle par la chaleur naturelle: aussi les embryons mâles sont-ils plus remuants que les embryons femelles. En se mouvant ils ont plus d'occasion de s'abîmer.» Quant aux femelles: «Il faut, dans le sein de la mère, plus de temps à la femelle qu'au mâle pour acquérir ses caractères distinctifs. Au contraire, une fois qu'elles sont nées, tout va chez les femelles plus vite vers son terme que chez les mâles, la puberté, l'âge mûr, la vieillesse. Car les femelles sont par nature plus faibles et plus froides, et il faut considérer leur nature comme une défectuosité naturelle.» ${ }^{53}$ Même le processus de l'accouchement diffère: dans l'Histoire des animaux, Aristote note que l'embryon mâle vient au monde plus vite que l'embryon femelle; les douleurs de l'enfantement sont plus continues et plus sourdes pour les filles, plus aiguës et plus pénibles pour les garçons ${ }^{54}$.

Toutes ces différences rendent la grossesse de jumeaux de sexes différents particulièrement compliquée: «La raison précitée explique également que chez l'homme les jumeaux de sexe différent ont moins de chance de survivre (...). Chez l'homme en effet, il est contraire à l'ordre naturel que les jumeaux mâles et femelles se développent au même rythme car la différenciation de leurs parties ne se produit pas en même temps: il est nécessaire que le mâle

49 Hist. an. 7, 4, 584 b. Voir aussi Probl. 894a 7-11.

50 Nat. 7, 37.

51 Cf. Orib. Coll. med. 22, 9.

52 Nat. puer. 18,1. Diogène d'Apollonie aurait précisé que «le corps d'un garçon met 4 mois à se former et celui d'une fille 5»; Cens. 9, 2 (= Diels, Vorsokr. 64 A 26).

53 Gen. an. 4, 6, 775a 4-16.

54 Hist. an. 7, 4, 584a 27-30. Cf. Soranos, Gynaecia, 1, 15. 
soit en retard ou la femelle en avance.» ${ }^{55} \mathrm{Ce}$ sont en somme de «véritables»

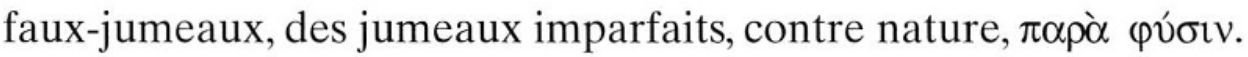

On peut discerner les traces de cette idée reçue dans les textes non médicaux relatifs au destin de paires fraternelles formées d'un jumeau et d'une jumelle, comme les enfants du dictateur Sylla, Faustus et Fausta, et ceux d'Antoine et Cléopâtre, Alexandre-Hélios et Cléopâtre-Sélénè ${ }^{56}$. Ces jumeaux ne constituent un couple que grâce à leurs noms. Quant il s'agit d'évoquer leurs destins, les auteurs anciens n'évoquent jamais leurs ressemblances ou leur complicité fraternelle, comme si leur différence de sexe n'en faisaient pas de véritables jumeaux ${ }^{57}$. La mythologie dénombre d'ailleurs très peu d'exemples de couples de frère et sœur jumeaux ${ }^{58}$. Ces paires sont encore plus rares dans l'iconographie. Apollon et Artémis sont parfois gémellisés, notamment dans l'iconographie étrusque. Un miroir provenant de la nécropole de Cerveteri ( $2^{\mathrm{e}}$ moitié du $5^{\mathrm{e}} \mathrm{s}$. av. J.-C.) montre ainsi Apollon attaquant avec sa sœur le serpent monstrueux qui pourchassait leur mère Léto (fig. 4). Mais ce document est inhabituel. Dans l'iconographie attique, seul le petit Apollon accomplit cet exploit; les imagiers du $5^{\mathrm{e}}$ s. av. J.-C. le montrent dans les bras de sa mère, se redressant pour viser le serpent ${ }^{59}$.

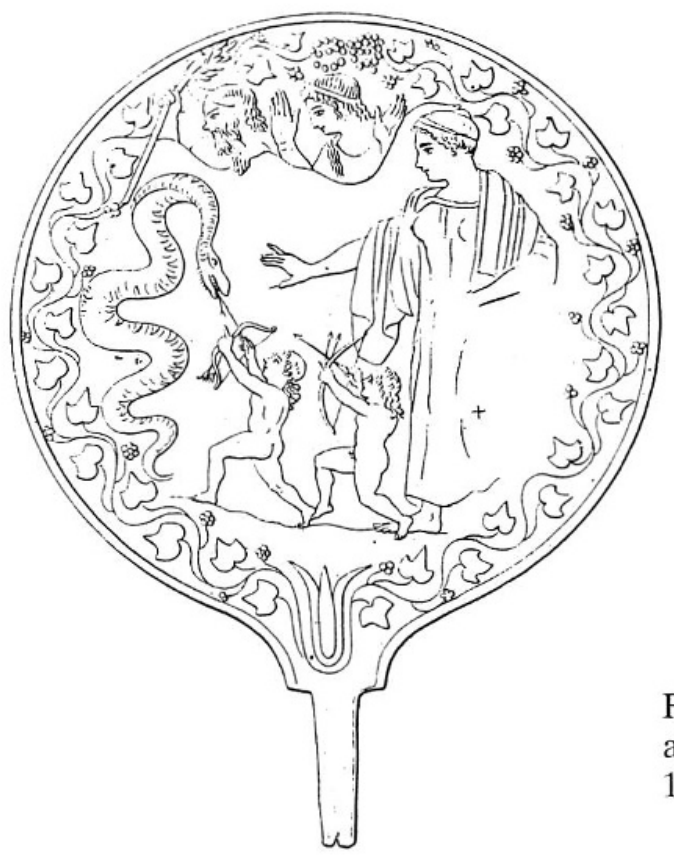

Fig. 4. Miroir étrusque (deuxième moitié du $5^{\mathrm{e}} \mathrm{s}$. av. J.-C.). Rome, Villa Giulia 51109. D'après LIMC II, $1984,339, \mathrm{n}^{\circ} 11$.

55 Gen. an. 4, 6, 775a 22-27.

56 Plut. Vie Syll. 34, 3; Vie Ant.36, 3.

57 Cf. F. Mencacci, I fratelli amici. La rappresentazione dei gemelli nella cultura romana, Venezia, 1996, 100.

58 Cf. les couples d'Amphiloque et Tisiphone, Byblis et Caunos, Cassandre et Hélénos, Narcisse et sa sœur.

59 W. Lambrinudakis et al., LIMC II, 1984, s.v. Apollon, 301-303, n ${ }^{\text {os } 988, ~ 993-4, ~ 998 ; ~ I . ~ K r a u s-~}$ kopf, ibid., s.v. Apollon/Aplu, 347, n $^{\text {os }} 79-84$. 


\section{Les conceptions gémellaires anormales}

\section{La superfétation}

Les Anciens ont reconnu l'existence d'un mode plus rare de conception gémellaire. Il s'agit de la superfétation, qui consiste en une deuxième fécondation au cours de la grossesse. Les auteurs en distinguent plusieurs formes selon l'intervalle de temps séparant les différentes conceptions: une superfétation peut avoir lieu immédiatement après la première conception ou pendant la grossesse ${ }^{60}$.

Lorsque la superfétation survient juste après la première fécondation, le deuxième embryon peut se développer et naître viable. Ce phénomène peut se produire lors d'un seul coït. Dans la Génération des animaux, Aristote explique que «dès qu'il y a plus d'un enfant, l'un est en superfétation par rapport à l'autre», car le sperme surabondant se divise et «produit plusieurs petits qui naissent les uns après les autres $»^{61}$. Cette idée est déjà présente chez l'auteur du traité De la Nature de l'enfant quand il note que le sperme est émis en plusieurs fois ${ }^{62}$. Erasistrate et les Stoïciens attribuent également les naissances gémellaires à ce phénomène; selon Pseudo-Galien, ils auraient soutenu que «lorsque le sperme tombe dans la première et deuxième régions, il se produit une superfétation et [des jumeaux ou] des triplés naissent ${ }^{63}$.

Mais deux fécondations distinctes peuvent aussi survenir lors de rapports sexuels avec deux partenaires différents; elles aboutissent alors à la naissance de deux enfants qui n'ont pas le même père. Dans l'Histoire des animaux, Aristote illustre cette théorie par le cas légendaire d'Héraclès et Iphiclès: la légende raconte que les deux enfants furent conçus par Alcmène à une nuit d'intervalle, Héraclès par Zeus, sous la forme d'Amphitryon, puis Iphiclès par Amphitryon de retour le jour suivant. Aristote décrit aussi le cas d'une femme qui mit au monde deux enfants dont l'un ressemblait à son mari, l'autre à son amant ${ }^{64}$. Pline rapporte des cas similaires, comme celui de cette servante volage qui, «suite à des relations dans la même journée, accoucha d'un enfant ressemblant au maître et d'un autre ressemblant à l'intendant ${ }^{65}$.

60 La terminologie moderne diffère si un deuxième ovocyte est fécondé au cours du même cycle (superfécondation) ou d'un cycle successif (superfétation); voir Papiernik-Berkhauer/Pons (n. 29 supra), 14 et 413; Phelan (n. 38 supra), 1047-1048. Pour la définition antique, C. Lienau, «Die Behandlung und Erwähnung von Superfetation in der Antike», Clio medica, 6, 1971, 275-285.

61 Gen. an. 4, 5,773b 7-12.

62 Nat. puer. 31, 3 (Littré VII 540).

63 De phil. hist. 32 (Kühn XIX 326) = Plut. Mor. 906B.

64 Hist. an. 7, 4, 585a 12-16.

65 Plin. Nat. 7, 49. 
En somme, le mari ne saurait être l'unique auteur de ces jumeaux dissemblables, alors qu'en réalité rien ne s'y opposerait! ${ }^{66}$ Mais l'hypothèse de l'adultère permettait d'expliquer les différences troublantes entre les jumeaux dizygotes. Notons qu'Aristote n'appelle pas ces enfants adultérins des jumeaux: dans l'Histoire des animaux, il précise que «les femmes portent le fruit supplémentaire et accouchent comme s'il s'agissait de jumeaux» ${ }^{67}$.

Le thème de la double paternité se retrouve dans la littérature non médicale. Un exemple étrange concerne Commode et Antonin, les fils jumeaux de Faustine la jeune et de Marc-Aurèle. On peut lire dans l'Histoire Auguste que des rumeurs d'adultère couraient sur les circonstances de la conception de Commode. Les uns disaient que Faustine était une épouse dévergondée qui aimait fréquenter les marins et les gladiateurs; elle se serait éprise d'un gladiateur dont elle aurait conçu (par superfétation?) Commode, «qui fut moins un empereur qu'un gladiateur». Mais pour les autres l'adultère ne fut pas réellement consommé. Une «petite histoire qui courait parmi le peuple» racontait que des astrologues conseillèrent à Marc-Aurèle de faire donner à sa femme un bain de siège dans le sang de son amant avant de s'unir à elle. Pensait-on que ce bain influença la conception des jumeaux, par une sorte d'imprégnation non par le regard mais par le sang $9{ }^{98}$ Le texte ne le précise pas, mais il est révélateur que l'on ait attribué à la mère de jumeaux un comportement sexuel déréglé. Comme si l'excès gémellaire devait correspondre à un excès sexuel, comme si la rupture de l'ordre naturel était liée à un désordre social.

Notons que le thème de la double paternité semble être resté du domaine de la spéculation médicale et de la rumeur populaire. A ma connaissance, aucun texte de loi antique n'évoque un tel cas, alors que le droit romain ne craint pas d'envisager les situations les plus rares et les plus insolites. L'éventualité de la double paternité aurait pourtant pu apparaître dans le cadre d'une succession litigieuse, mais les juristes romains ne l'ont jamais prise en considération.

Une seconde fécondation peut aussi se produire durant la grossesse. Hippocrate et Aristote notent que cette anomalie très rare ne survient qu'à la condition que la matrice de la femme enceinte ne se soit pas refermée com-

$66 \mathrm{Au}$ contraire, un phénomène de type superfétatoire pourrait expliquer une partie des conceptions gémellaires: selon M. G. Bulmer, The biology of twinning in man, Oxford, 1970, 82-83, une étude publiée en 1959 aurait montré que le taux de conception gémellaire est plus élevé chez les femmes enceintes au cours des premiers mois de mariage. Cf. Phelan (n. 38 supra), 1052-1053.

67 Hist. an. 7, 4, 585a.

68 H. A. Vie de Marc Antoine 19, 3 et 6 (trad. A. Chastagnol, Paris, Laffont, 1994). Mais M. Cornelius Fronto, Ep. 1, 3 vante leur ressemblance extraordinaire avec leur père. 
plètement et que les règles ne soient pas interrompues. Mais les conséquences de cette deuxième conception sont dramatiques. La superfétation se termine d'ordinaire par un avortement. Pour les auteurs hippocratiques, le

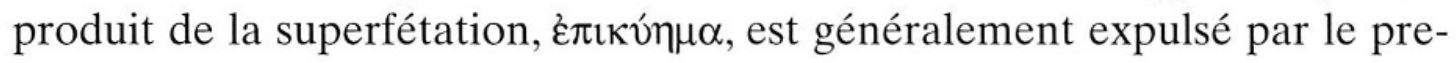
mier fœus ou naît prématurément en même temps que le premier enfant; il peut aussi nuire et même détruire le premier embryon ${ }^{69}$. Un cas est décrit dans les Epidémies: à Larissa une femme accoucha au neuvième mois d'une fillette vivante, mais avec une plaie sur la hanche; quarante jours plus tard «le produit d'une superfétation sortit, c'était une chair $(\sigma \alpha ́ p \xi))^{70}$.

Aristote suit la tradition hippocratique: «En tous cas les embryons formés longtemps après un autre ne viennent pas à terme, mais ils font du mal et amènent la destruction du précédent.» ${ }^{71} \mathrm{Il}$ rapporte aussi plusieurs cas de superfétation, comme celui de cette femme qui accoucha à terme de jumeaux bien constitués et d'un embryon de cinq mois qui mourut aussitôt ${ }^{72}$. Comme Hippocrate, Aristote s'abstient de nommer ces enfants superfétatoires des «jumeaux».

Cette théorie de la superfétation peut reposer sur l'observation de différents phénomènes réels. De manière générale, il n'est pas rare que les jumeaux présentent des inégalités de taille et de poids à la naissance qui ont pu faire croire à deux conceptions séparées dans le temps. Le cas décrit dans les Epidémies pourrait se rapporter à une complication relativement fréquente des grossesses gémellaires, la mort in utero d'un des jumeaux. Chez le jumeau survivant, cet accident peut entraîner des lésions importantes (viscérales, rénales, cérébrales...), voire aboutir à un accouchement prématuré; l'aspect du fœtus mort-né macéré a pu être confondu avec celui d'un embryon de quelques mois ${ }^{73}$. En outre, il n'est pas impossible que les Anciens aient effectivement observé des jumeaux d'âges différents conçus par superfétation; la réalité de ce phénomène exceptionnel a été récemment établie scientifiquement ${ }^{74}$.

La théorie de la superfétation donne une image plutôt négative de la gémellité. En effet, la plupart des animaux refusent l'accouplement pendant

69 Superf. 1 (= Littré VIII 476); Vict. 1, 31 (Littré VI 506). Voir aussi Aristot. Gen. an. 4, 5, 773b 13-18.

70 Epid. 5, 11 (= Littré V 210-212).

71 Hist. an. 7, 4, 585a 8-10. Cf. Gen. an. 4, 5, 773b 16-18.

72 Hist. an. 7, 4, 585a 16-19.

73 Papiernik-Berkhauer/Pons (n. 29 supra), 67-70 (syndrome transfuseur-transfusé, siamois, foetus in foetu), 167-176 (mort in utero d'un jumeau); Phelan (n. 38 supra), 1058-1063.

74 Voir p.ex. P. Bourgoin et al., «A propos d'un cas de grossesse gémellaire dizygotique où la superfoetation paraît peu contestable», J. Gynecol. Obstet. Biol. Reprod., 24, 1995, 440-443 ( 2 embryons à 3 et 6 semaines de différence). 
la grossesse, à l'exception de la jument et de la hase, des animaux réputés pour leur lascivité. La jument accepte le coït à cause de son tempérament sensuel, mais elle ne peut pas concevoir un deuxième embryon ${ }^{75}$. La fécondité exceptionnelle de la hase est liée à sa nature hypersexuée. Son cycle reproductif est ininterrompu, la superfétation est son mode normal de conception: «Les lièvres (...) s'accouplent et mettent bas en toute saison; les femelles sont sujettes à la superfétation et mettent bas tous les mois.» ${ }^{76} \mathrm{Chez}$ l'être humain, la naissance de jumeaux peut ainsi apparaître comme l'indice d'un comportement sexuel excessif, empreint d'animalité; plus grave encore, la mère de jumeaux dissemblables peut être soupçonnée d'adultère.

\section{Les jumeaux monstrueux}

Aristote l'affirme à plusieurs reprises: l'idéal est de n'avoir qu'un enfant à la fois. Comme il l'explique dans la Génération des animaux, un excès de matière séminale peut produire soit des jumeaux distincts, soit des êtres monstrueux avec un organe ou une partie surnuméraire: «Quant à la formation contre nature de parties en surnombre, elle a la même cause que la production des jumeaux. Cette cause se trouve déjà chez les embryons, si la matière qui prend forme est plus abondante que ne l'exige la nature de la partie à former.» ${ }^{77}$ Un être double peut aussi se créer par manque d'espace dans l'utérus. La présence d'un ou plusieurs cœurs sert alors de critère pour reconnaître «si un animal monstrueux est un animal unique, ou s'il est composé de plusieurs animaux soudés ensemble» ${ }^{78}$. Les prémices de cette théorie se trouvent chez Empédocle et Démocrite qui mettent également en cause un surplus de matière séminale et sa division anormale pour expliquer la formation d'êtres monstrueux ${ }^{79}$.

Aristote apporte de nouveaux éléments en se basant sur l'observation d'œufs de poule. Il remarque que cet animal ovipare et multipare produit souvent des petits anormaux «parce qu'elles (les poules) ont plusieurs produits à la fois et qu'elles s'accouplent en toute saison $»^{80}$. Aristote constate

75 Hist. an. 7, 4, 585a 3-7; Gen. an. 4, 5, 773b 25-35. Voir aussi Elien Nat. 4,11. Plin. Nat. 10, 182 ajoute la truie.

76 Hist. an. 6, 2, 579b 31-33. Cf. Gen. an. 4, 5, 774 a 31-774b 4. Voir aussi p.ex. Hdt. 3, 108, Plin. Nat. 8, 219.

77 Gen. an. 4, 4, 772b 13-26.

78 Gen. an. 4, 4,773a 8-12.

79 Démocrite ap. Aristot. Gen. an 4, 3, 769b 30-34 (Diels, Vorsokr. 68 A 146); Empédocle ap. Plut. Mor. 905E-906A (= H. Diels, Doxographi Graeci, Berlin, 1879, 420,19-421, 5) et Ps.Gal. De phil. hist. 32, Kühn XIX 326). Cf. Ch. G. Bien, Erklärungen zur Entstehung von Missbildungen im physiologischen und medizinischen Schriftum der Antike, Stuttgart, 1997, 67-74. 80 Gen. an. 4, 4, 770a 10-13. 


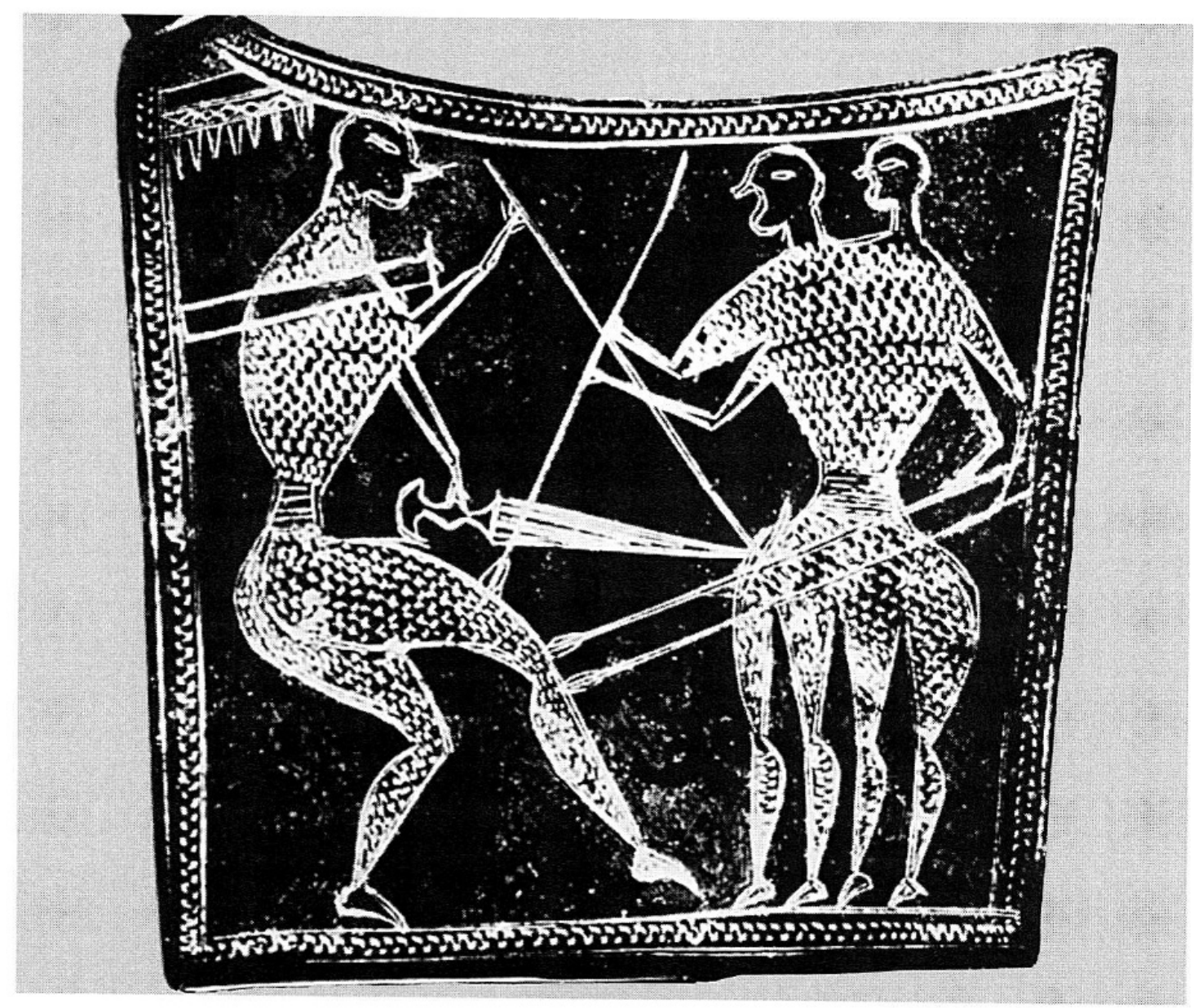

Fig. 5. Fibule en bronze (début du $7^{\mathrm{e}}$ s. av. J.-C.). Athènes, Musée national 11765. D'après $L I M C$ I, 1981, pl. 364, n 7 .

aussi que certaines poules ne pondent que des œufs à deux jaunes, et que sur une ponte de dix-huit œufs doubles éclôt un poussin monstrueux ${ }^{81}$. Ses observations sont même très précises. Il note: «Lorsque les blancs sont séparés par la membrane, il naît deux poussins distincts qui n'ont rien de particulier. Mais quand ils sont contigus et que rien ne les sépare, il naît des poussins monstrueux, avec un seul corps et une seule tête, mais quatre pattes et quatre ailes (...).» ${ }^{82}$ Le même raisonnement explique que ce type d'anomalie ne se produit jamais chez les abeilles et les guêpes, «car leurs œufs sont déposés dans des cellules séparées» ${ }^{83}$.

En se référant à l'observation d'œuf de poules, Aristote met à nouveau en relation la multiparité et l'animalité. Le thème des jumeaux issus d'un œuf

81 Hist. an. 6, 3, 562a 24-562b 2.

82 Gen. an. 4, 4, 770a 18-23. Cf. Probl. 898a 17-18. Le serpent à deux têtes est un autre exemple d'ovipare monstrueux: Gen. an. 4, 4, 770a 23-25.

83 Gen. an. 4, 4, 770a 27-29. 
revient souvent dans la mythologie classique. Plusieurs récits concernent des êtres doubles. Les Molionides (ou Actorides), Eurytos et Ctéatos, seraient ainsi nés d'un œuf d'argent, leurs deux corps soudés l'un à l'autre. Ils auraient même deux pères différents, Poséidon et Actor, l'époux de Molioné. Les textes ne donnent toutefois pas de détails sur les circonstances de leur conception et de leur naissance ${ }^{84}$. Les imagiers de l'époque géométrique ont représenté ces jumeaux avec un réalisme étonnant. Une fibule en bronze du début du $7^{\mathrm{e}} \mathrm{s}$. av. J.-C. montre ainsi les Molionides en lutte contre Héraclès; ils sont figurés avec deux têtes, quatre bras et quatre jambes émergeant d'un tronc commun (fig. 5). Mais ces images de jumeaux siamois sont exceptionnelles dans l'art grec. Elles n'apparaissent qu'à la fin de l'époque géométrique, du milieu du $8^{\mathrm{e}} \mathrm{s}$. au début du $7^{\mathrm{e}} \mathrm{s}$. av. J.-C. Les Molionides sont ensuite complétement absents des arts figurés.

Phanès (Protogonos ou Eros), le dieu de la lumière primordiale dans la théogonie orphique, naît aussi d'un œuf d'argent, pondu par la Nuit; cet

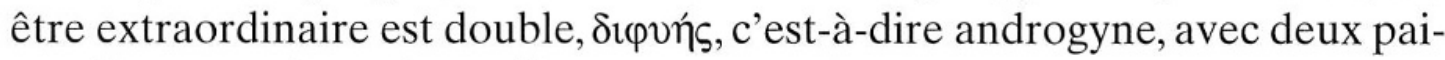
res d'yeux ou deux visages ${ }^{85}$.

D'autres couples gémellaires sont associés aux œufs. Les Dioscures, Castor et Pollux, naissent également d'un œuf avec leur sœur Hélène. Leur légende connaît plusieurs variantes. Ils sont soit issus de l'union de Némésis transformée en oie avec Zeus sous la forme d'un cygne; l'œuf de Némésis est alors recueilli par Léda. Ou bien c'est Léda elle-même qui est l'amante du cygne divin et qui pond un ${ }^{86}$ ou deux œufs, l'un contenant Clytemnestre et Pollux, l'autre Castor et Hélène ${ }^{87}$, ou l'un avec Castor et Clytemnestre, l'autre avec Pollux et Hélène ${ }^{88}$.

Si l'œuf ne contient qu'Hélène pour les imagiers grecs, il renferme des triplés, les Dioscures et Hélène, pour les artistes d'époque romaine. Plusieurs documents représentent cette naissance extraordinaire. Un sarcophage en marbre du $2^{\mathrm{e}}$ s. apr. J.-C., conservé à Aix-en-Provence (fig. 6), illustre les principaux épisodes de l'histoire de Léda. A gauche, la jeune femme nue

84 Pour les sources littéraires et iconographiques, voir R. Hampe, LIMC I, 1981, s.v. Aktorione, 472-476; V. Dasen, «Des Molionides à Janus: les êtres à corps ou à parties multiples dans l'Antiquité classique», in H.-K. Schmutz (éd.), Phantastische Lebensräume, Phantome und Phantasmen, Marburg an der Lahn, 1997, 120-122. Sur leur naissance d'un œuf, Ibycos ap. Athen. 57f-58a (D. L. Page, Poetae Melici Graeci, Oxford, Clarendon Press, 1962, fr. 4).

85 O. Kern, Orphicorum fragmenta, Berlin, 1922, fr. 1, 70, 87 (œuf); 76, 77 (quatre yeux ou deux faces), 81, 87 et 98 (bisexué). Cf. L. Brisson, Orphée et l'orphisme dans l'Antiquité grécoromaine, Aldershot/Brookfield, 1995, 2885.

86 Premier mythographe du Vatican, 1, 77. Cf. Paus. 3,16, 1 sur l'œuf (des Dioscures?) exposé dans le sanctuaire des Leucippides à Sparte.

87 Premier mythographe du Vatican, 3, 1, 64.

88 Apollod. 3, 10, 7; Hyg. Fab. 77. 


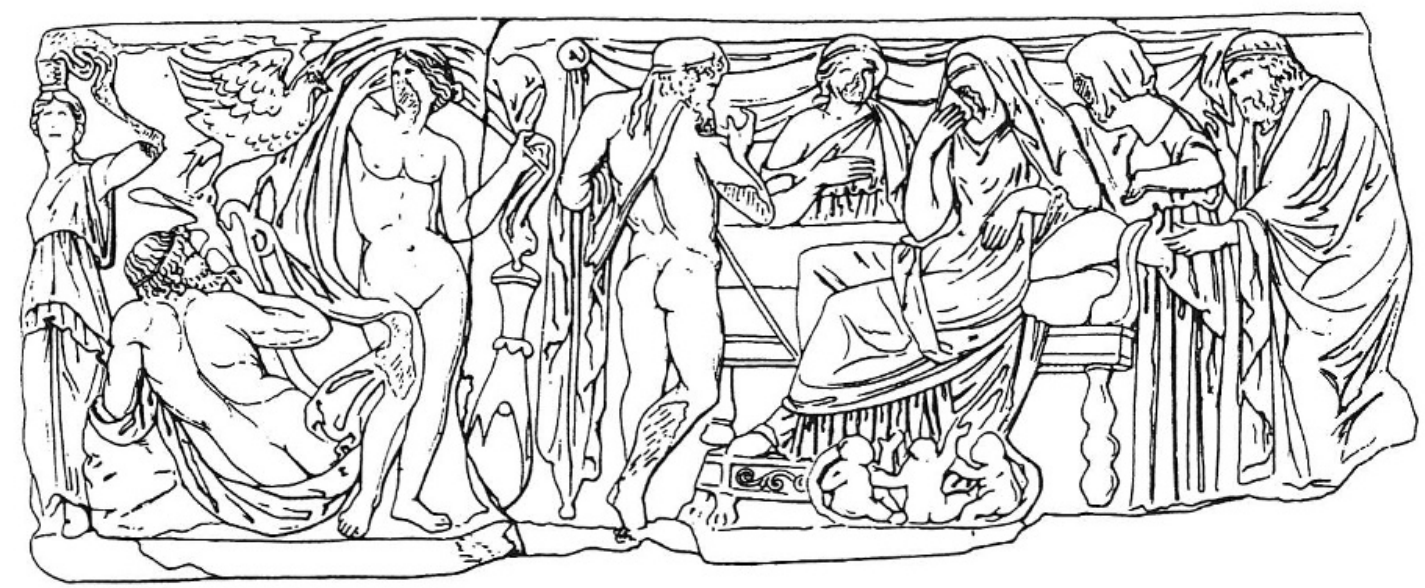

Fig. 6. Sarcophage de marbre, Aix-en-Provence, Musée Granet ( $2^{\mathrm{e}} \mathrm{s}$. apr. J.-C.). D'après LIMC IV, 1988, 504, n 11.

s'apprête à s'unir au cygne; à droite, elle regarde, assise sur un lit, la naissance des triplés qui émergent de la coquille brisée de l'œuf ${ }^{89}$.

De nombreux auteurs anciens assimilent les bonnets coniques des Dioscures (piloi) aux deux moitiés de coquille de cet œuf origine $1^{90}$. Mais la signification de cet œuf ne saurait être réduite à des considérations biologiques et tératologiques. Il possède aussi une dimension cosmique et fait allusion au caractère astral des deux frères. Pour les orphiques, les piloi des Dioscures symbolisent la terre et le ciel, les deux moitiés de l'œuf primordial pondu par la Nuit. Pour les Pythagoriciens, ces bonnets étoilés représentent les deux moitiés de la sphère céleste, passant alternativement du jour à la nuit; l'union parfaite des deux frères symbolise l'harmonie de l'univers ${ }^{91}$.

Le thème de l'œuf apparaît aussi dans la littérature non médicale à propos de couples gémellaires non monstrueux. On compare volontiers les jumeaux à deux gouttes de lait ou à deux œufs. Pour démontrer les limites de leurs similitudes, Cicéron, dans la première Académique, utilise ainsi l'image des œufs: «Une mère, par exemple, distingue ses jumeaux l'un de l'autre, ses yeux sont instruits par l'accoutumance: toi-même tu les distingueras si tu les vois habituellement. Te rappelles-tu que la ressemblance des œufs a passé en proverbe? Nous savons cependant qu'à Délos, au temps de sa prospérité,

89 L. Kahil, LIMC IV, 1988, s.v. Helene, 503-4, n ${ }^{\text {os }} 1-10$ (Hélène seule); 504, n ${ }^{\text {os }} 11-13$ (Hélène et ses frères).

90 Ce chapeau devient leur attribut régulier dès le $3^{\text {e }}$ s. av. J.-C. Cf. Lycophron, Alexandra, 506-507; Lucien, Dial. deor. 25, 1 (éd. M. D. Macleod, Stuttgart, Teubner, 1987).

91 F. Cumont, Recherches sur le symbolisme funéraire des romains, Paris, 1942, 69-70; F. Gury, LIMC III, 1986, s.v. Dioskouroi/Castores, 610. Le motif des jumeaux primordiaux issus d'un ou de deux œufs se retrouve dans d'autres cultures. Cf. S. M. S. Carvalho, «Soleil et lune: les jumeaux mythiques et le caractère tricheur", in Les grandes figures religieuses. Fonctionnement pratique et symbolique dans l'Antiquité, Besançon 25-26 avril 1984, Paris, 1986,159-164. 
beaucoup de personnes nourrissaient des poules pour en faire commerce et, à l'inspection d'un œuf, savaient dire quelle poule l'avait pondu.» ${ }^{92}$

\section{Conclusion}

La réflexion sur les naissances gémellaires a permis aux Anciens de préciser diverses théories sur la génération, notamment sur les conditions nécessaires à la conception, au développement du fœetus et à la détermination des sexes. L'intérêt de ces théories déborde du cadre de l'histoire des sciences. Leur trait le plus frappant est l'opposition des vues de l'école hippocratique et d'Aristote. Pour les auteurs hippocratiques, la naissance de jumeaux est un événement exceptionnel, mais dans l'ordre naturel. Pour Aristote, ces naissances sont classées parmi les anomalies. Les jumeaux de sexes différents forment une catégorie à part: ils apparaissent comme des jumeaux imparfaits, contre nature, tandis que les jumeaux superfétatoires sont le produit de la conduite impudique, empreinte d'animalité, de leur mère, parfois soupçonnée d'adultère ${ }^{93}$. Cette ambivalence se retrouve dans l'opinion commune. Dans l'iconographie et les textes non médicaux, les naissances gémellaires sont tantôt accueillies comme des signes d'abondance et de fécondité, tantôt associées à des notions de souillure et d'illégitimité.

\section{Remerciements}

Les conclusions de cet article ont été présentées au séminaire de médecine antique organisé par V. Barras et Ph. Mudry à l'Université de Fribourg en juin 1998. Je tiens aussi à remercier pour ses remarques Danielle Gourevitch (Paris, Ecole Pratique des Hautes Etudes, IV ${ }^{\mathrm{e}}$ section) qui a relu une première version de cet article.

\section{Abréviations}

Diels, Vorsokr. = H. Diels/W. Kranz, Die Fragmente der Vorsokratiker, 3 vol., Berlin, ${ }^{6} 1951$.

Kühn = Galien, Opera omnia, éd. et trad. lat. C. G. Kühn, 20 vol., Leipzig, 1821-1833.

Littré $\quad=$ Hippocrate, Opera omnia, éd. et trad. fr. E. Littré, 10 vol., Paris, 1839-1861.

LIMC = Lexicon Iconographicum Mythologiae Classicae, Zürich/München, I-VIII, 1981-1997.

Auteurs anciens: les titres sont abrégés selon les conventions de W. Sontheimer/K. Ziegler (éds), Der Kleine Pauly. Lexikon der Antike in fünf Bänden, I, München, 1975.

Sauf mention contraire, toutes les traductions citées ici sont tirées des éditions Belles-Lettres à Paris.

92 Ac. 1 (Lucullus), 18 (trad. C. Appuhn, Paris, éd. Garnier). Répété par Galien, De placitis Hippocratis et Platonis 9, 9 (Kühn V 802); Adm. an. 3, 5 (Kühn II 384).

93 Cette ambivalence se retrouve chez les auteurs modernes. Certains ne retiennent que les éléments positifs des textes médicaux relatifs aux jumeaux; cf. F. Frontisi-Ducroux, «Les Grecs, le double et les jumeaux», Topique, 50,1992,240-242. D'autres, au contraire, les interprètent de manière négative; cf. Mencacci (n. 56 supra), 14-37. L'ouvrage de Stevenson/Hall/Goodman (n. 38 supra) suit l'école d'Aristote en incluant les naissances gémellaires dans son inventaire des anomalies chez l'homme. 